\title{
X-rays from accretion shocks in classical T Tauri stars: 2D MHD modeling and the role of local absorption
}

\author{
C. Argiroffi ${ }^{1,2}$, R. Bonito ${ }^{1}$, S. Orlando ${ }^{2}$, M. Miceli ${ }^{2}$, F. Reale Re $^{1,2}$, \\ G. Peres ${ }^{1,2}$, T. Matsakos ${ }^{3,4}$, C. Sthelé ${ }^{5}$ and L. Ibgui ${ }^{5}$ \\ ${ }^{1}$ Dip. di Fisica e Chimica, Università di Palermo, Palermo, Italy, email: \\ argi@astropa.unipa.it; ${ }^{2}$ INAF - Osservatorio Astronomico di Palermo, Piazza del \\ Parlamento 1, 90134 Palermo, Italy; ${ }^{3}$ CEA, IRAMIS, Service Photons, Atomes et Molécules, \\ Gif-sur-Yvette, France; ${ }^{4}$ Laboratoire AIM, CEA/DSM - CNRS - Université Paris Diderot, \\ IRFU/SAp, Gif-sur-Yvette, France; ${ }^{5}$ LERMA, Observatoire de Paris, Universitè Pierre et \\ Marie Curie, CNRS, 5 place J. Janssen, Meudon, France.
}

\begin{abstract}
In classical T Tauri stars (CTTS) strong shocks are formed where the accretion funnel impacts with the denser stellar chromosphere. Although current models of accretion provide a plausible global picture of this process, some fundamental aspects are still unclear: the observed X-ray luminosity in accretion shocks is order of magnitudes lower than predicted; the observed density and temperature structures of the hot post-shock region are puzzling and still unexplained by models.

To address these issues we performed 2D MHD simulations describing an accretion stream impacting onto the chromosphere of a CTTS, exploring different configurations and strengths of the magnetic field. From the model results we then synthesized the X-ray emission emerging from the hot post-shock, taking into account the local absorption due to the pre-shock stream and surrounding atmosphere.

We find that the different configurations and strengths of the magnetic field profoundly affect the hot post-shock properties. Moreover the emerging X-ray emission strongly depends also on the viewing angle under which accretion is observed. Some of the explored configuration are able to reproduce the observed features of X-ray spectra of CTTS.
\end{abstract}

Keywords. Accretion, MHD, Stars: pre-main sequence, X-rays: stars

\section{MHD simulations}

CTTS are young low mass stars still accreting material from their circumstellar disks. Accreting material forms strong shock impacting on the stellar surface, being therefore heated by these shocks up to $\sim 1-5 \mathrm{MK}$, and emitting significantly in the X-rays. High resolution X-ray spectra of CTTS provide us several observational constraints on the accretion process. However some fundamental aspects, like the over-predicted $L_{\mathrm{X}}$ from accretion shocks, and the observed density vs temperature structures of the hot postshock region, are puzzling and still unexplained by models.

To address these issues we performed 2D MHD simulations of the accretion shock region (fig. 1), using the PLUTO code (Mignone et al. 2007), exploring different configurations and strengths of the stellar magnetic field (Orlando et al. 2013). For each simulation we computed the total emerging X-ray spectrum produced by the hot post-shock plasma, taking into account the different absorption suffered by X-rays emitted by different postshock portions, and the different possible viewing angles. 


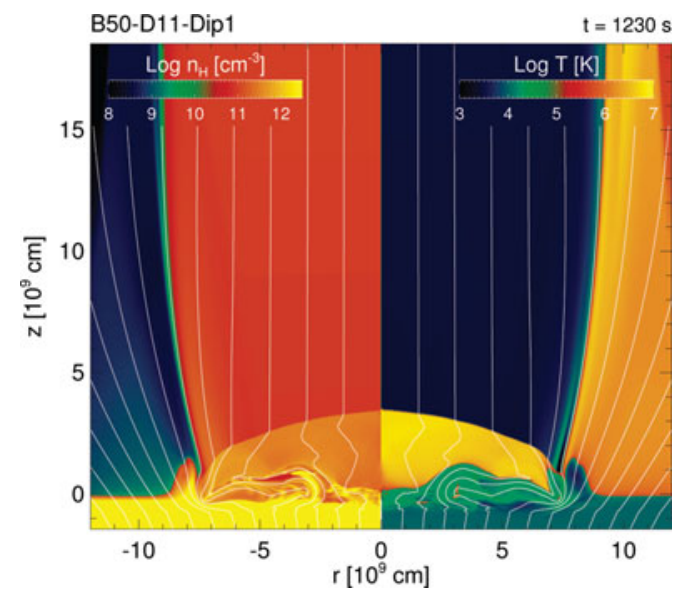

Figure 1. Density (left panels) and temperature (right panels) distributions of one of the simulations; the white lines mark magnetic field lines.

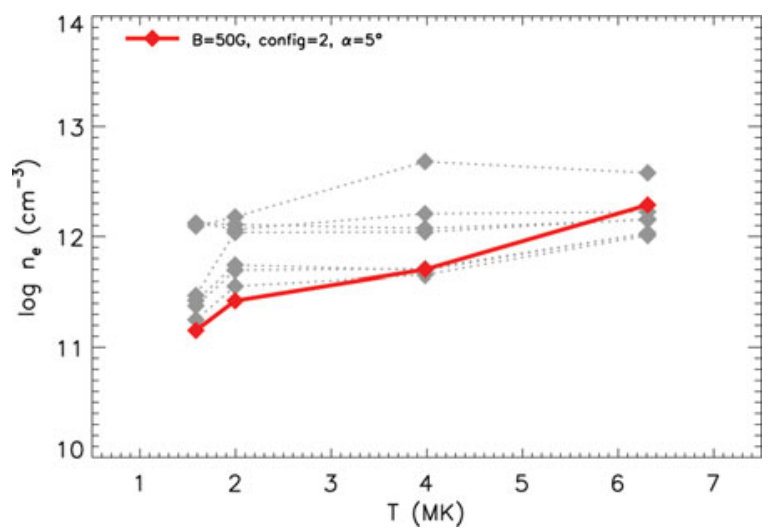

Figure 2. Densities vs temperature obtained from the He-like triplets diagnostics of the emerging X-ray spectra corresponding to different simulations and different viewing angles.

\section{Results}

We find that both magnetic field strength and configuration deeply affect the location and characteristics of post-shock plasma, and, as a consequence, affect the absorption suffered by the emitted X-rays. The emerging X-ray spectra for different cases and for different viewing angles strongly differ in shape and luminosity. In particular we find that some configurations produce X-ray emerging spectra that reproduce the density vs temperature pattern (fig. 2) observed in the X-ray spectra of CTTS, and up to date not explained by models.

On the one hand our results indicate that accretion shock models are able to reproduce the features observed in the X-ray band of CTTS. On the other hand our results evidence how magnetic field configuration, local absorption, and viewing angle are fundamental parameters to interpret the observed X-ray emission from accretion shock in CTTS.

\section{References}

Mignone, A., Bodo, G., Massaglia, S., Matsakos, T., Tesileanu, O., Zanni, C., \& Ferrari, A. 2007, ApJS, 170, 228

Orlando, S., Bonito, R., Argiroffi, C., et al. 2012, A\&A, in press 